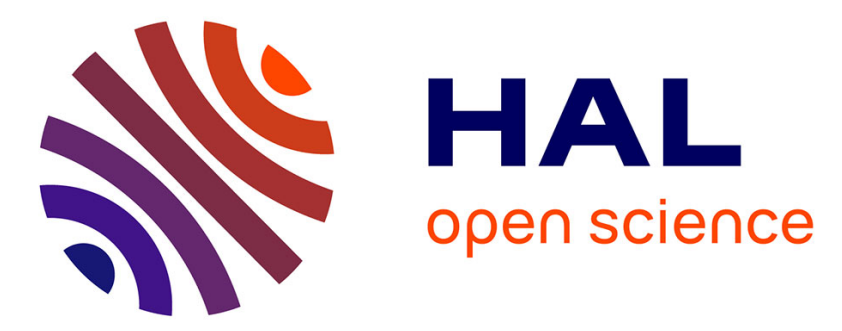

\title{
Structure of velocity distributions in shock waves in granular gases with extension to molecular gases
}

Alexandre Vilquin, J. F. Boudet, H. Kellay

\section{To cite this version:}

Alexandre Vilquin, J. F. Boudet, H. Kellay. Structure of velocity distributions in shock waves in granular gases with extension to molecular gases. Physical Review E , 2016, 94 (2), pp.022905 (1-14). 10.1103/PhysRevE.94.022905 . hal-01392711

\section{HAL Id: hal-01392711 \\ https://hal.science/hal-01392711}

Submitted on 4 Nov 2016

HAL is a multi-disciplinary open access archive for the deposit and dissemination of scientific research documents, whether they are published or not. The documents may come from teaching and research institutions in France or abroad, or from public or private research centers.
L'archive ouverte pluridisciplinaire HAL, est destinée au dépôt et à la diffusion de documents scientifiques de niveau recherche, publiés ou non, émanant des établissements d'enseignement et de recherche français ou étrangers, des laboratoires publics ou privés.

\section{(1)(1) $\$(0)$}

Distributed under a Creative Commons Attribution - NonCommercial - ShareAlikel 4.0 


\title{
Structure of velocity distributions in shock waves in granular gases with extension to molecular gases
}

\author{
A. Vilquin, J. F. Boudet, and H. Kellay \\ Université de Bordeaux, Laboratoire Ondes et Matière d'Aquitaine, UMR 5798 U. Bordeaux/ \\ CNRS, 351 cours de la Libération, 33405 Talence, France
}

\begin{abstract}
Velocity distributions in normal shock waves obtained in dilute granular flows are studied. These distributions cannot be described by a simple functional shape and are believed to be bimodal. Our results show that these distributions are not strictly bimodal but a trimodal distribution is shown to be sufficient. The usual Mott-Smith bimodal description of these distributions, developed for molecular gases, and based on the coexistence of two subpopulations (a supersonic and a subsonic population) in the shock front, can be modified by adding a third subpopulation. Our experiments show that this additional population results from collisions between the supersonic and subsonic subpopulations. We propose a simple approach incorporating the role of this third intermediate population to model the measured probability distributions and apply it to granular shocks as well as shocks in molecular gases.
\end{abstract}

\section{INTRODUCTION}

Unlike a sound wave, which is a small-amplitude compression wave leaving the propagation medium unchanged, a shock wave creates violent changes in pressure, velocity, density, and temperature on short length scales comparable to the mean free path [1]. In the reference frame of the shock wave, the supersonic gas is compressed and heated in the front region to become subsonic on the other side. The shock front structure (see Fig. 1) has been the subject of several studies in gases [2-8], plasmas [9-11], and in granular materials [12-19]. A normal shock wave was first described as a discontinuity. The conditions linking the macroscopic properties on either side of the shock front were deduced by V. Rankine (1870) and H. Hugoniot (1889) and several theoretical studies [20-23] focused on such properties.

A conspicuous feature of shock waves is their highly nonequilibrium nature. In 1951 Mott-Smith developed a theory [20] where a shock wave is described as a superposition of a supersonic stream and a subsonic stream: their proportions vary with position along the front and a bimodal velocity distribution is assumed. Figure 1 illustrates this theory with the density increase in the shock front along with the probability density functions (PDF) of the velocity at different positions along the front. The thin Gaussian PDF of the supersonic stream (A) is gradually replaced by the wider Gaussian PDF in the heated subsonic stream (C). A superposition of these two distributions and thus a bimodal distribution is assumed in the shock front (B). This model provided a basis for calculating shock wave properties such as the shock width and gave rise to many subsequent studies [23-26].

Despite its fundamental importance, the bimodal structure of the velocity distributions had to await experimental confirmation. Experiments in different media [5,6,9,10,17] show that the velocity distributions cannot be described by a simple functional shape in the shock front region. Measurements of the velocity distributions in the shock front [6] in gases showed that the velocity PDFs are not strictly bimodal pointing to the existence of a substantial number of particles with intermediate velocities (see Fig. 1). A few studies [27,28] reproduce these experimental PDFs with molecular dynamics as well as Monte Carlo simulations. However, and despite their key role in understanding the shock front structure, particle velocity distributions are still poorly understood.

In this paper, we examine the velocity distributions in normal shock waves obtained in dilute granular flows. These flows have similarities with gas flows [29-36] and are the basis for the development of the granular kinetic theory constructed from the inelastic Boltzmann equation. The low speed of granular pressure disturbances (typically in the range of $\mathrm{cm} / \mathrm{s}$ ), equivalent to the speed of sound in gases, allows us to easily generate shock waves [13-17]. Here, we use two experimental setups to produce granular shock fronts. The first is a dilute granular flow around a circular obstacle as in Ref. [17], where a bimodal-like structure for the velocity distributions has been observed. The second is a vibrated granular gas where the shock front is produced by a moving rectangular obstacle and for which a bimodal-like structure is again observed. While these velocity distributions can be described qualitatively using a bimodal distribution, a substantial amount of particles have intermediate velocities and do not belong to either the supersonic or the subsonic stream as observed in gases. Our main interest is the origin of these particles and our experiments show that the particles with intermediate velocities are the result of only a few collisions between the incident supersonic stream and the subsonic stream. A simple model where the two streams interact in the shock front region via binary collisions allows us to predict the density of these intermediate particles and therefore the velocity distributions at different locations along the shock front.

The article is organized as follows. First we recall the properties of shock fronts in gases and summarize the theory of Mott-Smith for the velocity distributions. We then describe our experiments using gravity-driven granular flows around a circular obstacle as well as the identification and analysis of the intermediate particle population. This leads us to propose a model to obtain this population from binary collisions between the supersonic and subsonic streams. We use this model to analyze another set of experiments using a vibrated granular 

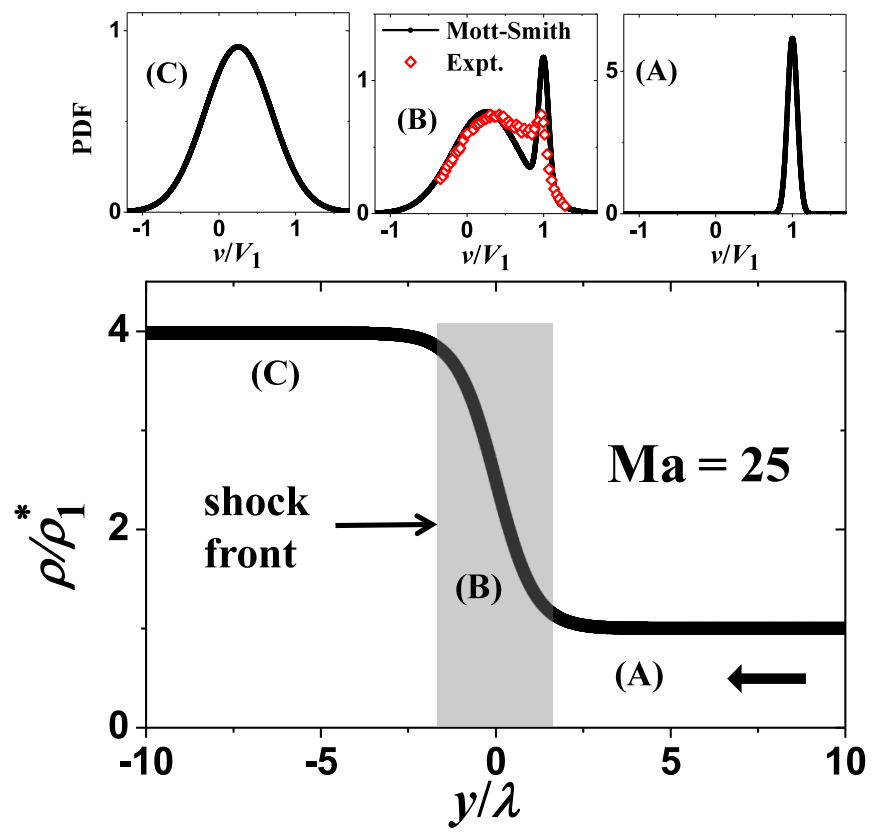

FIG. 1. Density profile in a normal shock wave with PDFs of particle velocity in the insets, based on the Mott-Smith theory for $\mathrm{Ma}=25$. Experimental data in the inset (B) come from Ref. [6]. Note the presence of a significant number of particles with intermediate velocities not accounted for by the Mott-Smith distribution.

gas and other experimental and numerical results obtained using molecular gases.

\section{SHOCK FRONTS IN MOLECULAR GASES}

In this section, we recall the properties of the velocity distributions in a one-dimensional shock front. First, let us introduce the jump relations established by Rankine and Hugoniot. These relations, obtained by using the conservation laws for energy, momentum, and mass through the shock front for a nondissipative gas, can be written in the reference frame of the shock front (for a perfect gas with $n$ degrees of freedom) as

$$
\begin{aligned}
\frac{\rho_{2}^{*}}{\rho_{1}^{*}} & =\frac{n+1}{1+n \mathrm{Ma}^{-2}}, \\
\frac{V_{2}}{V_{1}} & =\frac{1+n \mathrm{Ma}^{-2}}{n+1}, \\
\frac{T_{2}}{T_{1}} & =\frac{\left(1+n \mathrm{Ma}^{-2}\right)\left[(n+2) \mathrm{Ma}^{2}-1\right]}{(n+1)^{2}} .
\end{aligned}
$$

These relations link the values of the mean velocity $V_{2}$, the temperature $T_{2}$, and the number density $\rho_{2}^{*}$ (far from the front) describing the subsonic flow to their counterparts describing the supersonic flow $\left(V_{1}, T_{1}, \rho_{1}^{*}\right)$ for different Mach numbers, which is defined as $\mathrm{Ma}=V_{1} / C_{1}$, where $C_{1}$ is the sound speed in the supersonic flow. These relations give no indication as to the density profile or the temperature profile across the shock front. This information requires a detailed description of the front region.

To describe the shock front, Mott-Smith separates the shock into two subpopulations, the supersonic and subsonic populations, which exist on either side of the front, and as- sumes a superposition of these two subpopulations in the front region. He then makes a very simple hypothesis; the velocity distribution in the shock front is bimodal and is the sum of two Gaussian distributions reflecting the coexistence of these two populations in the shock front. The velocity and temperature of each population are those before and after the shock front (and interconnected by the jump relations above), but the proportion of each population varies across this front. The velocity distribution function is then written as $f=f_{1}+f_{2}$ :

$$
f_{i}(\vec{c})=\frac{\rho_{i}(y)}{\left(2 \pi k T_{i} / m\right)^{3 / 2}} \exp \left(-\frac{\left(\vec{c}-V_{i} \overrightarrow{e_{y}}\right)^{2}}{2 k T_{i} / m}\right), \quad i=1,2 .
$$

Here $\vec{c}=u \overrightarrow{e_{x}}+v \overrightarrow{e_{y}}+w \overrightarrow{e_{z}}$ is the vector velocity of a particle, $k$ and $m$ are the Boltzmann constant and the mass of the molecules, respectively, and $y$ is the coordinate in the direction perpendicular to the shock front. The number densities $\rho_{i}(y)$ represent, respectively, the local density of supersonic particles $(i=1)$ and subsonic particles $(i=2)$. To find the density profile across the shock, Mott-Smith then calculates the moment of order 2 of the longitudinal velocity $v$ using the Boltzmann equation [20] and obtains the following closure equation:

$$
\frac{d \rho_{1}(y)}{d y}=-B \frac{\rho_{1}\left(1-\rho_{1} / \rho_{1}^{*}\right)}{\lambda},
$$

where $\lambda$ is the mean free path. The coefficient $B$ only depends on Ma and $n$. This equation is used to find the number density profile of each population:

$$
\begin{aligned}
& \rho_{1}(y)=\rho_{1}^{*}[1+\exp (-B y / \lambda)]^{-1}, \\
& \rho_{2}(y)=\rho_{2}^{*}[1+\exp (B y / \lambda)]^{-1} .
\end{aligned}
$$

The width of the shock front is therefore given by $L_{\mathrm{MS}}=$ $4 \lambda / B$. The bimodal distribution proposed by Mott-Smith is, however, not a solution of the Boltzmann equation, which describes the shock front (a similar calculation with the third moment, instead of the second moment of the longitudinal velocity, leads to a similar closure equation but with a coefficient $B$, which is slightly different). Nonetheless, the results obtained using this theory are very similar to those observed in experiments and simulations. For example, for a gas of hard spheres, the shock width variation with the Mach number complies with numerical simulations to within $10 \%$ accuracy. In fact, the results given by Mott-Smith quite faithfully reflect macroscopic variables and their evolution through the shock front. But, they do not accurately reflect the shape of the velocity distributions; along the longitudinal axis, the experimentally measured distributions show that there is an overpopulation at intermediate velocities (see Fig. 1) hinting at the existence of an additional population.

Salwen [24] extended the Mott-Smith approach to a threepopulation model. He added a third population to the two populations $f_{i}(i=1,2)$ given by Mott-Smith to take into account the surplus at intermediate velocities. He selected a form for the velocity distribution of the third population $(i=3)$ that satisfies the Euler equations written for a model with three 

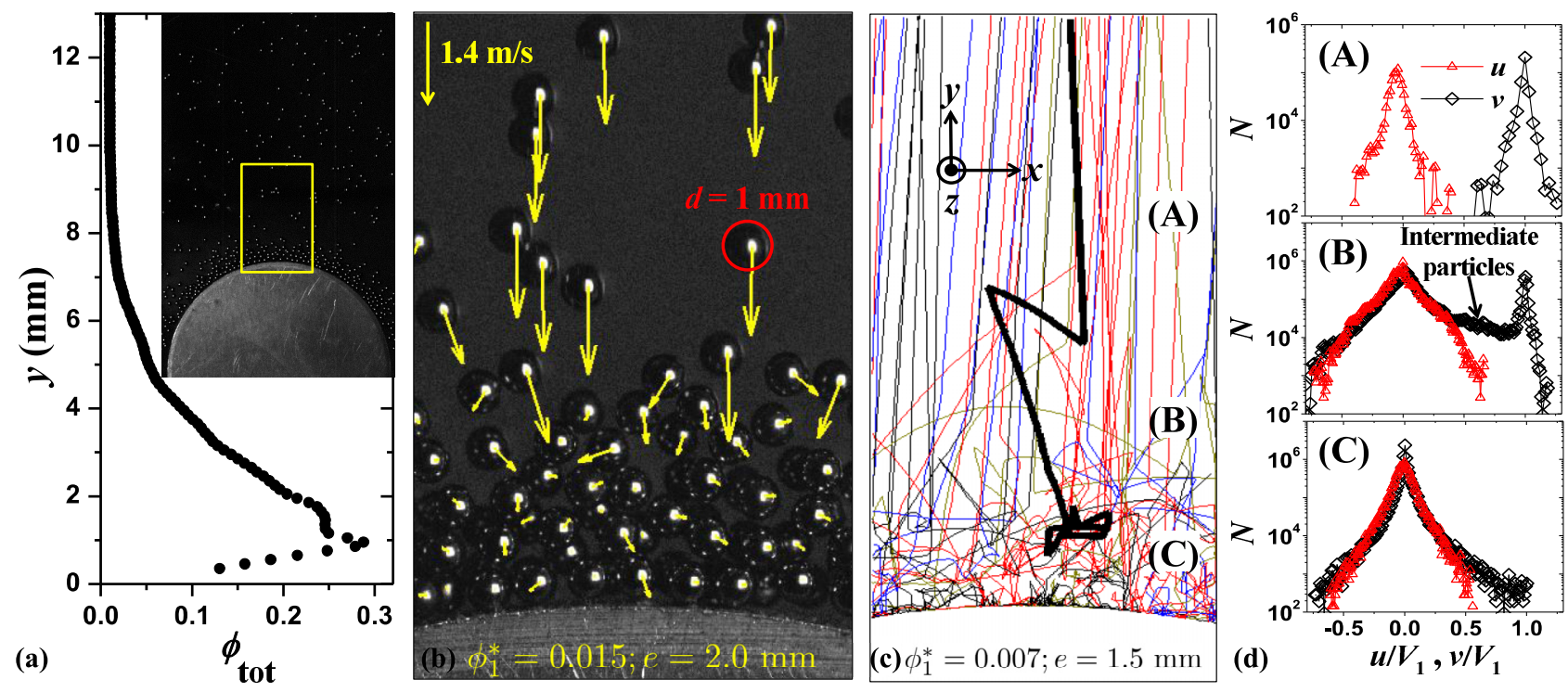

FIG. 2. (a) Total volume fraction $\phi_{\text {tot }}$ in the shock front plotted versus the distance $y$ from the obstacle. Note that the particle density is high near the obstacle and decreases as the distance to the obstacle increases. 0 corresponds to the summit of the circular obstacle. Inset: photograph of the shock front induced around a circular obstacle. The yellow rectangle indicates the study area (b), where $\phi_{\text {tot }}$ is measured. (a), (b), $e=2 \mathrm{~mm}, \phi_{1}^{*}=0.015, V_{1}=1.4 \mathrm{~m} / \mathrm{s}$, and Ma $\sim 60$. (b) Photograph of the shock front around a circular obstacle in the study area, shown in the inset of (a). Here the obstacle is at the bottom of the image and is light gray while the particles appear as dark disks with a small bright spot near the middle. Yellow arrows indicate particle velocities. Both photographs [inset in (a) and (b)] are taken using reflection from a broad white light source. (c) Representation of 50 particle paths, with one highlighted in bold, in a normal shock front in a granular flow around a circular obstacle. (d) Histograms of $v$ (black) and $u$ (red), respectively, longitudinal and transverse velocities normalized by the mean velocity of the supersonic stream $V_{1}$ in three different regions: the initial stream (A), the shock front (B), and near the obstacle (C). Note the presence of intermediate velocity particles in region (B). (c), (d), $e=1.5 \mathrm{~mm}, \phi_{1}^{*}=0.007, V_{1}=1.1 \mathrm{~m} / \mathrm{s}$, and $M \sim 30$.

populations (written here for $\mathrm{Ma}=\infty$ and $n=3$ ):

$$
\begin{aligned}
f_{3}(\vec{c}) & =\frac{\rho_{3}(y)}{\left(2 \pi k T_{3} / m\right)^{3 / 2}}\left|\frac{v-V_{3}}{\sqrt{k T_{3} / m}}\right| \exp \left(-\frac{\left(\vec{c}-V_{3} \overrightarrow{e_{y}}\right)^{2}}{2 k T_{3} / m}\right), \\
V_{3} & =V_{1} / 2 \quad \text { and } T_{3}=m V_{1}^{2} / 20 k .
\end{aligned}
$$

Then he calculated two higher-order moments of the velocity using the Boltzmann equation to get two closure relations, which are needed for a model with three populations. However, this distribution is not in agreement with the experimental distributions; in particular, $f_{3}=0$ for $v=V_{1} / 2$, which is not born out experimentally (see Fig. 1). In this work, we highlight the importance of the third population as well as point out its origin from experiments in granular shock fronts.

\section{SHOCK WAVES IN DILUTE GRANULAR FLOWS AROUND AN OBSTACLE}

\section{A. Experiments and results}

In this section we use a granular gas flowing around an obstacle to produce shock fronts and examine the velocity distributions across this front in detail. As mentioned above, the system we use shows the presence of a bimodal-like distribution for the velocity in the shock front region [17]. We examine here the presence of intermediate velocity particles in this region and track their origin through the tracking of individual particles as they enter the shock front.

The experimental setup uses two parallel glass plates set vertically and spaced by a distance $e(1.5 d<e<8 d)[12,17]$.
Steel beads $(d=1 \mathrm{~mm})$ are poured between the glass plates from an upper reservoir equipped with a gate to control the incident volume fraction of the particles. A circular obstacle (radius $R=4 \mathrm{~cm}$, thickness $e$ ) is inserted between the plates at distances between 5 and $30 \mathrm{~cm}$ from the cell entrance. The beads fall under the action of gravity. The circular shape of the obstacle forces a two-dimensional flow [inset Fig. 2(a)] and a stationary shock front is formed above the obstacle summit. The total particle volume fraction $\phi_{\text {tot }}$ increases from low values near the incident volume fraction far from the obstacle to much higher values near the obstacle. The volume fraction of the incident supersonic stream $\phi_{1}^{*}$ can be varied from $0.4 \%$ to $2 \%$. Its velocity $V_{1}$ can be varied from 1 to $2.5 \mathrm{~m} / \mathrm{s}$.

To obtain the local volume fractions and the particle velocities, the reflected light from the beads is collected with a fast camera at 4000 frames/s, allowing an efficient counting and tracking of the particles [Fig. 2(b)]. Each bead acts as a spherical mirror reflecting light from a spot smaller than the bead diameter. This makes the tracking of particles and their collisions simple as during a collision between two beads, the light spots stay separated [see Fig. 2(b)]. From the granular temperature $T=\delta c^{2}(\delta \vec{c}$ is the standard deviation of the velocity $\vec{c}$ [29]) and $\phi_{1}^{*}$, we estimate the Mach number Ma for this flow configuration to be between 10 and 60 depending on the mean velocity and the temperature of the incident stream. This velocity depends on the position of the obstacle with respect to the entrance, while the temperature depends on this position as well as the entrance conditions. Both quantities are systematically measured in each experiment. The speed of sound used here, taking 6 degrees of freedom, 
is given by [37]

$$
c^{\prime}=\sqrt{T X\left[1+\frac{1}{3} X+\frac{\phi}{X} \frac{d X}{d \phi}\right]},
$$

where $X(\phi)=1+2(1+r) \phi /\left(1-\left(\phi / \phi_{\mathrm{MAX}}\right)^{\frac{4}{3} \phi_{\mathrm{MAX}}}\right), r$ is the inelasticity coefficient and $\phi_{\mathrm{MAX}}=0.65$ is the random closepacking volume fraction.

The starting point for understanding the velocity distributions in the shock front above the obstacle is summarized in Fig. 2. This figure shows both particle paths [Fig. 2(c)] and the histograms of particle velocities denoted $N$ [Fig. 2(d)]. Three different regions can be distinguished. In region (A), particles falling under gravity in the supersonic stream do not collide with each other. The velocity histogram has a single sharp peak centered at the free fall velocity $V_{1}$. In region (B), which corresponds to the shock front, the particles experience only a few collisions and an overall bimodal-like shape of the velocity histogram is observed as in Ref. [17]. In region (C), the density of particles increases and numerous collisions occur; the velocity histograms are single peaked but broad giving a higher granular temperature than in region (A). These observations show that the flow is constituted by a supersonic stream (region A), a subsonic stream where the temperature is higher and the particle collisions are numerous (region $\mathrm{C}$ ), and an intermediate region where collisions are sparse but the histograms look bimodal. As in gases [6], an important fraction of particles with intermediate velocities is observed. The key to understanding the velocity histograms and therefore the velocity PDFs (obtained by a proper normalization of the histograms) is to relate their shapes to the occurrence of collisions. By studying particle paths [see Fig. 2(c)], we can disentangle particles that have experienced no collisions from particles that have experienced 1,2 , or more collisions in regions (B) and (C) and gauge their role and weight in the velocity distributions.

In Fig. 3, we plot as open symbols the volume fraction of detected particles that have experienced no collisions $\phi_{1}$ versus distance from the obstacle $y$ normalized by the mean free path in the incident stream $\lambda=d / 6 \sqrt{2} \phi_{1}^{*}$. Note that $\phi_{1} / \phi_{1}^{*}$ is near 1 far from the obstacle, decreases as collisions start to occur in region (B) before going to zero in region (C), where the number of collisions becomes important. Figure 3 also shows the volume fraction of detected particles having experienced a single collision $\phi_{3}$ as well as two collisions $\phi_{4}$. Both fractions are near zero in region (A) where no collisions occur, increase in region (B), and go through a peak before decreasing to zero in region $(\mathrm{C})$. Note that $\phi_{3}$ has a peak at an earlier position than $\phi_{4}$. While we tracked particles up to 3 collisions, successive collisions are increasingly difficult to detect as they occur in regions where the total volume fraction $\phi_{\text {tot }}$ increases (see inset of Fig. 3). The other symbols (solid lines and solid symbols) are discussed below.

In order to illustrate this further, we show in Fig. 4 the PDFs of $v$ (the component of the velocity normal to the shock front) for particles following 1,2, and 3 collisions obtained from particle tracking [see Fig. 2(a)]. These PDFs are for the velocities obtained just after collision. Note that particles with a single collision have higher velocities and should contribute the most to the presence of intermediate

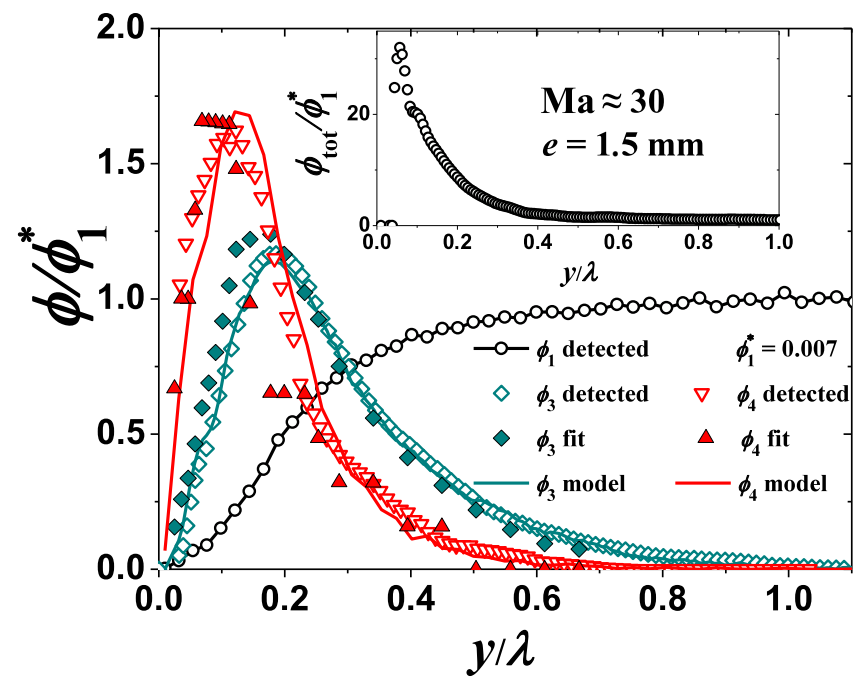

FIG. 3. Volume fractions $\phi_{1}(y), \phi_{3}(y)$, and $\phi_{4}(y)$ of supersonic, first-generation, and second-generation intermediate particles, respectively. The open symbols are measurements obtained from direct tracking (see Sec. III A). The solid lines are from our model where $A_{32} / A_{12}=1.05$ and $A_{42} / A_{12}=1.4$ (see Sec. III B). The solid symbols are from the fit of the velocity PDFs with four populations (as shown in Fig. 6). The total volume fraction is shown in the inset. $\left(e=1.5 \mathrm{~mm}, \phi_{1}^{*}=0.007, V_{1}=1.1 \mathrm{~m} / \mathrm{s}\right.$, and $\left.M \sim 30\right)$.

velocities in the full distribution of $v$ shown in Fig. 2 since the velocity PDFs of particles with 2 and 3 collisions have a peak at lower speed close to zero. Figure 4 also shows

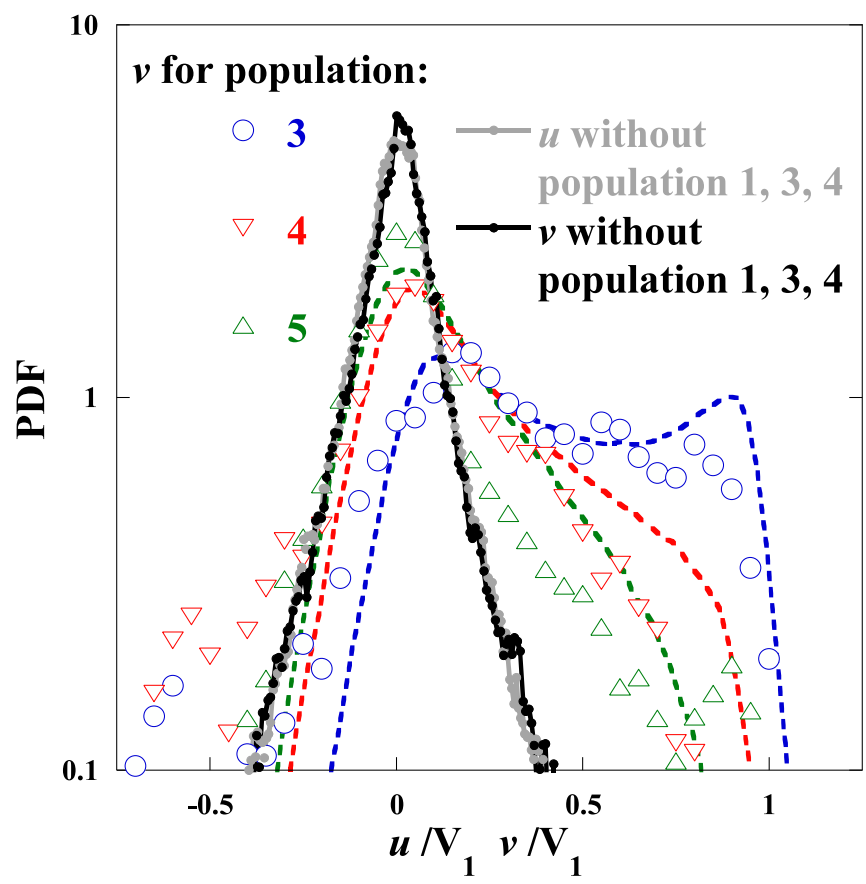

FIG. 4. PDFs of $v$ obtained just after 1, 2, or 3 collisions along with the PDFs of $u$ and $v$ from which the contribution of the supersonic as well as the histograms of particles after 1 or 2 collisions has been subtracted. Note the isotropy of the velocity once this subtraction is carried out. The PDFs of the subsonic particles will be identified with these latter distributions. 

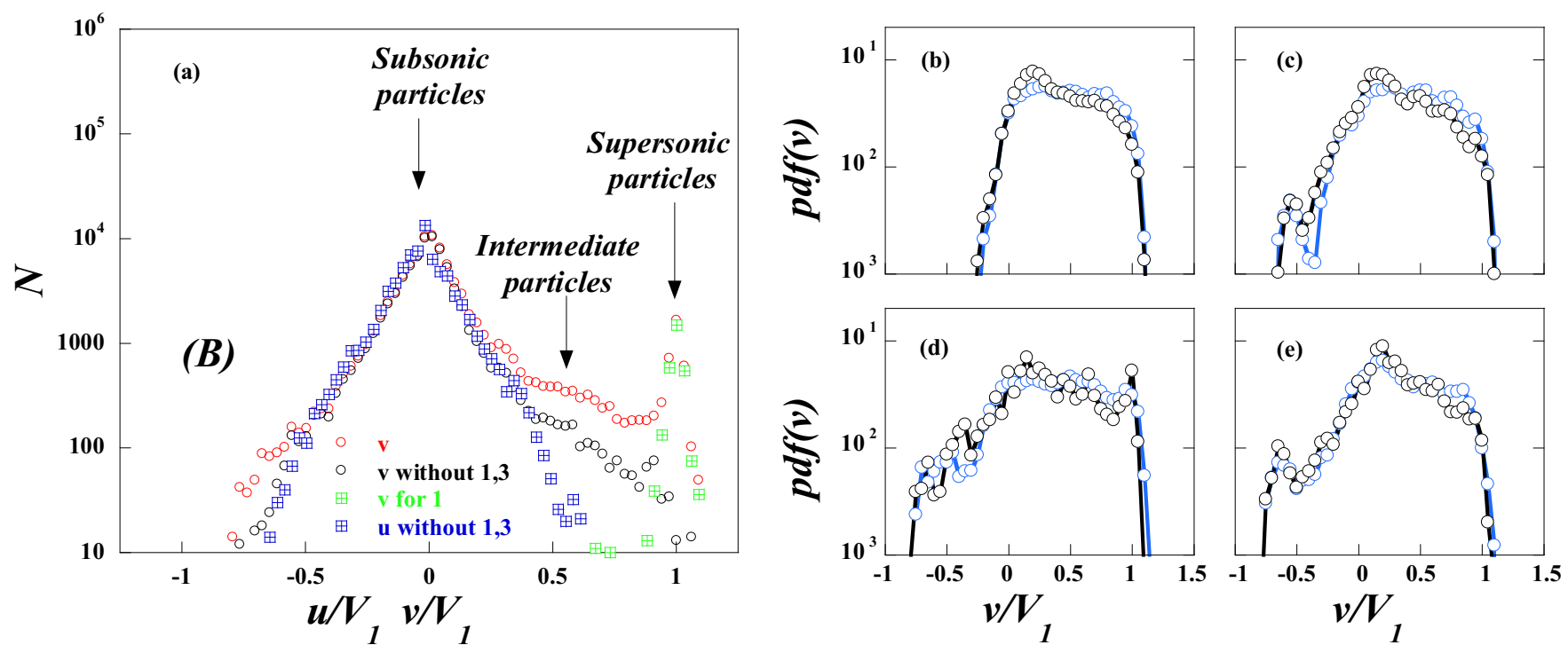

FIG. 5. (a) Histograms of $u$ and $v$, normalized by the mean velocity of the supersonic stream $V_{1}$ in region (B). The different symbols represent the histograms of $v$ for all particles (red), for supersonic particles only (green), $u$ (blue), and $v$ (black) without the contribution of intermediate particles with 1 collision. Note that subtracting the contribution of the supersonic particles and that from intermediate particles render the histograms of $v$ closer to that of $u$ by suppressing an important proportion of intermediate particles. The subtraction of the contribution from intermediate particles to the PDF of $u$ does not change its shape visibly $\left(e=1.5 \mathrm{~mm}, \phi_{1}^{*}=0.007, V_{1}=1.1 \mathrm{~m} / \mathrm{s}\right.$, and $M \sim 30$ ). While in (a) we have subtracted the contribution of the histogram of the full intermediate particle population (for 1 collision), (b)-(e) show that the histograms of this intermediate population (black symbols) obtained in region (B) are well approximated by the histograms of particle velocities obtained just after a single collision (blue symbols). (b) $e=2 \mathrm{~mm}, \phi_{1}^{*}=0.022, V_{1}=1.5 \mathrm{~m} / \mathrm{s}$. (c) $e=2 \mathrm{~mm}, \phi_{1}^{*}=0.004, V_{1}=1.5 \mathrm{~m} / \mathrm{s}$. (d) $e=1.5 \mathrm{~mm}, \phi_{1}^{*}=0.007, V_{1}=1.1 \mathrm{~m} / \mathrm{s}$. (e) $e=4 \mathrm{~mm}, \phi_{1}^{*}=0.007, V_{1}=1.5 \mathrm{~m} / \mathrm{s}$.

the PDF of $u$ as well as the PDF of $v$ to which we have subtracted the contribution from the supersonic stream (i.e., particles having experienced no collisions) and from particles with two collisions or less. For this subtraction, we have used the measured volume fractions shown in Fig. 3. An important observation is that these latter PDFs are roughly similar: the velocity is isotropic once the contribution from particles with few collisions has been removed. This points to the fact that the population of particles with intermediate velocities is mainly the result of a few collisions between the incident supersonic stream and the subsonic stream near the obstacle. While the supersonic stream can be identified with its sharp velocity distribution at the incident stream velocity and also obtained from particle tracking experiments by identifying the particles having experienced no collisions, the subsonic stream is more difficult to extract and we identify this subsonic stream with the isotropic velocity distributions obtained by subtracting the contribution from a few collisions as discussed above and shown in Fig. 4. In fact, and in order to reconstruct the subsonic stream distribution, a measurement of the distribution of $u$ to which the contribution of the supersonic stream is subtracted turns out to be sufficient since the additional subtraction of particles with a few collisions does not induce measurable changes of this distribution. The distribution of $v$ is then taken as that of $u$. To illustrate this in detail, let us now go back to the full histogram of $v$ measured in region (B) and shown in Fig. 5(a). We use the histograms, i.e., the number of particles $N$, instead of probability density functions here, as we need to remove the contribution of certain classes of particles. The measured histogram has three main contributions: the supersonic stream with particles having experienced no collisions and whose histogram is known from the tracking experiments described above and which is superimposed on the full histogram, an isotropic subsonic stream with particles having experienced more than two or three collisions whose histogram of $v$ is similar to that of the transverse velocity $(u)$, and intermediate particles having experienced one or two collisions. Figure 5(a) shows that subtracting, from the histogram of $v$, the contribution of full intermediate particle population having experienced a single collision (as obtained from particle tracking experiments at the specific position from the obstacle) removes an important proportion of the intermediate velocity population. This graph indicates, nonetheless, that other intermediate populations with more than a single collision are needed to remove the full intermediate population.

Note here that the intermediate population needed to understand these distributions, obtained at a specific position from the obstacle as used in Fig. 5(a), is not equivalent to that obtained just after the collisions (as shown for the purpose of illustration in Fig. 4), since this latter does not take into account the intermediate particles advected into and out of the region of interest and does not take into account the disappearance of these particles from this region. However, and by tracking the true intermediate particles in a few runs, i.e., by tracking the particles over the whole experimental region and identifying the particles having experienced 1 collision and that have crossed this region, left it or were created within it (a full counting of all intermediate particles after 1 collision and just before a second collision), it turns out that the two distributions are close to each other as shown in Figs. 5(b)-5(e) for different volume fractions, incident 
velocities, and values of $e$. While we do not have a justification for this observation, the range of velocities that is of interest must be correct as both distributions are the result of the same collision between fast and slow particles. Further, the effects of advection and additional collisions act differently on the fast particles (more sensitive to advection which makes them leave the measurement volume) and the slow particles (more sensitive to a second collision and therefore disappear with a higher probability than the faster particles). In short, collisions act less on the faster particles and advection acts less on the slower particles. We speculate that the combination of the two makes for some compensation of the ignored effects in our approximation making for a functional shape that is close to that estimated using the creation part only.

Now that we have identified the origin of the intermediate population, what is needed to predict the full velocity distribution is a model for the density and velocity distribution of these particles. Here, we assume, in accordance with our observations, that these particles are the result of a few collisions between the supersonic and subsonic streams. We identify the supersonic stream with the peak at the incident velocity while the subsonic stream is identified as the isotropic part of the full distribution. We then adopt simple rules: A supersonic particle is converted to an intermediate particle after a collision with a subsonic particle. This subsonic particle is also converted to an intermediate particle. We also assume that these intermediate particles, which are the product of the first collision, will have shocks with subsonic particles only and are converted to second generation intermediate particles (the product of the second collision). We will call the first generation of intermediate particles population 3 and the second generation population 4 .

\section{B. Calculation of the volume fraction of intermediate particles}

Following Mott-Smith, let us describe a normal shock wave as a superposition of an incident supersonic stream (population 1), a subsonic stream (population 2), and a third state where particles have intermediate velocities (population 3). We assume this intermediate state to be the result of a single collision between supersonic particles and subsonic particles. We detail here the calculation of the volume fraction for one intermediate state (population 3); however, the approach can be extended to four subpopulations in a similar manner (see Appendix A).

We consider here three subpopulations: the supersonic, the subsonic, and the intermediate particles. Population transfers between these three subsets occur in the shock wave. Supersonic particles collide once with subsonic ones to become intermediate particles. In turn, these intermediate particles collide with existing subsonic particles, leading to other subsonic particles. We neglect collisions between intermediate particles and supersonic particles, which are far less numerous than the subsonic particles in the shock wave (in agreement with our experimental observations for the gravity-driven flow of particles around an obstacle). This assumption allows a great simplification in our model but is not a priori valid, generally. Thus, transfers for each subpopulation $i(=1,2$, or 3$)$ can be described by appearance and disappearance processes, which we write as

$$
\frac{\partial \phi_{i}}{\partial t}+\frac{\partial\left(\phi_{i} V_{i}\right)}{\partial y}=\left(\frac{\partial \phi_{i}}{\partial t}\right)_{\mathrm{disp}}+\left(\frac{\partial \phi_{i}}{\partial t}\right)_{\mathrm{app}} .
$$

Here $\phi_{i}$ and $V_{i}$ are, respectively, the particle volume fraction and the mean velocity along $y$ for the subpopulation $i$. For a stationary flow, $\frac{\partial \phi_{i}}{\partial t}=0$ for each subpopulation $i$. Supersonic, subsonic, and intermediate populations are, respectively, noted with an index $i$ taking the values 1,2, and 3, respectively. From our model, supersonic particles can only disappear after collisions with subsonic particles. One subsonic particle disappears per such collision:

$$
\frac{\partial\left(\phi_{1} V_{1}\right)}{\partial y}=\left(\frac{\partial \phi_{1}}{\partial t}\right)_{\text {disp }}=-J_{12}
$$

where $J_{12}$ is the collision operator between supersonic and subsonic particles. For two subpopulations $a$ and $b$,

$$
J_{a b}=\iint \Omega f_{a}\left(\vec{c}_{a}\right) f_{b}\left(\vec{c}_{b}\right)\left|\vec{c}_{a}-\vec{c}_{b}\right| d \vec{c}_{a} d \vec{c}_{b}
$$

$\Omega=\pi d^{2}$ is the cross section for hard spheres (particle diameter $d)$ and $f_{i}\left(\vec{c}_{i}\right)$ is the PDF of particle velocities, assumed Gaussian (for simplicity) in this calculation:

$$
f_{i}\left(\vec{c}_{i}\right)=\frac{\phi_{i}(y)}{\left(2 \pi T_{i}\right)^{3 / 2}} \exp \left(-\frac{\left(\vec{V}_{i}-\vec{c}_{i}\right)^{2}}{2 T_{i}}\right), \quad i=a, b .
$$

$T_{i}$ is the granular temperature for the subpopulation $i$. The collision operator can be written

$$
\begin{aligned}
J_{a b} & =J_{b a}=\frac{6 \sqrt{2}}{d} \phi_{a} \phi_{b}\left|V_{a}-V_{b}\right| \times A_{a b} \\
A_{a b} & =\frac{F^{3 / 2}}{6 \sqrt{\pi}} \int_{0}^{\infty}\left(|1+x|^{3}-|1-x|^{3}\right) \exp \left(-\frac{F x^{2}}{2}\right) x d x \\
F & =\frac{\left(V_{a}-V_{b}\right)^{2}}{T_{a}+T_{b}}
\end{aligned}
$$

Note that intermediate particles do not have a Gaussian velocity distribution in molecular gases, leading to a slightly different value of $A_{a b}$. Similarly, velocity distributions are not Gaussian in granular gases so the value of $A_{a b}$ given above is only indicative. Subsonic particles appear after collisions between intermediate particles and existing subsonic particles. They disappear by collisions with supersonic particles:

$$
\begin{aligned}
\frac{\partial\left(\phi_{2} V_{2}\right)}{\partial y} & =\left(\frac{\partial \phi_{2}}{\partial t}\right)_{\text {disp }}+\left(\frac{\partial \phi_{2}}{\partial t}\right)_{\mathrm{app}} \\
& =\left(\frac{\partial \phi_{2}}{\partial t}\right)_{\text {disp }}-\left(\frac{\partial \phi_{3}}{\partial t}\right)_{\text {disp }} \\
& =-J_{12}+J_{32} .
\end{aligned}
$$

Intermediate particles appear after collisions between supersonic particles and subsonic particles. A collision between a supersonic particle and a subsonic particle leads to two intermediate particles. They disappear after a second collision 
with a subsonic particle:

$$
\begin{aligned}
\frac{\partial\left(\phi_{3} V_{3}\right)}{\partial y} & =\left(\frac{\partial \phi_{3}}{\partial t}\right)_{\text {disp }}+\left(\frac{\partial \phi_{3}}{\partial t}\right)_{\text {app }} \\
& =\left(\frac{\partial \phi_{3}}{\partial t}\right)_{\text {disp }}-\left(\frac{\partial \phi_{1}}{\partial t}\right)_{\text {disp }}-\left(\frac{\partial \phi_{2}}{\partial t}\right)_{\text {disp }} \\
& =-J_{32}+2 J_{12} .
\end{aligned}
$$

Note that Eqs. (7), (12), and (13) respect the conservation of mass. Because of momentum and energy conservation, the mean velocity and the temperature of intermediate particles can be expressed as

$$
V_{3}=\frac{V_{1}+V_{2}}{2} \quad \text { and } \quad T_{3}=\frac{T_{1}+T_{2}}{2}+\frac{\left(V_{1}-V_{2}\right)^{2}}{4 n} .
$$

From Eqs. (7), (8), (13), and (14), we can rewrite

$$
\frac{\partial\left(\phi_{3} V_{3}\right)}{\partial y}=-2 \frac{\partial\left(\phi_{1} V_{1}\right)}{\partial y}-\frac{6 \sqrt{2}}{d} \phi_{3} \phi_{2}\left(V_{3}-V_{2}\right) \times A_{32} \text {. }
$$

The subsonic particle volume fraction $\phi_{2}$ can be expressed from Eq. (7):

$$
\frac{6 \sqrt{2}}{d} \phi_{2}=-\frac{1}{A_{12}} \frac{1}{V_{1}-V_{2}} \frac{1}{\phi_{1}} \frac{\partial\left(\phi_{1} V_{1}\right)}{\partial y} .
$$

Thus, we can express the intermediate particle flux as

$$
\frac{\partial\left(\phi_{3} V_{3}\right)}{\partial y}=-2 \frac{\partial\left(\phi_{1} V_{1}\right)}{\partial y}+\frac{1}{2} \frac{A_{32}}{A_{12}} \frac{\phi_{3}}{\phi_{1}} \frac{\partial\left(\phi_{1} V_{1}\right)}{\partial y} .
$$

The mean velocity of supersonic particles has a constant value. Also, we use an approximation where the mean velocity of intermediate particles varies slowly for high values of $V_{1}$. We then obtain

$$
\frac{V_{3}}{V_{1}} \frac{\partial \phi_{3}}{\partial \phi_{1}}=-2+\frac{1}{2} \frac{A_{32}}{A_{12}} \frac{\phi_{3}}{\phi_{1}} .
$$

With $\lim _{y \rightarrow \infty} \phi_{1}(y)=\phi_{1}^{*}$, we obtain the analytical solution for Eq. (18):

$$
\begin{aligned}
\phi_{3} & =K_{1} \phi_{1}^{*}\left[\frac{\phi_{1}}{\phi_{1}^{*}}-\left(\frac{\phi_{1}}{\phi_{1}^{*}}\right)^{\alpha}\right], \\
K_{1}^{-1} & =\frac{1}{2} \frac{V_{3}}{V_{1}}(\alpha-1), \\
\alpha & =\frac{1}{2} \frac{A_{32}}{A_{12}} \frac{V_{1}}{V_{3}} .
\end{aligned}
$$

Notice that this calculation is carried out for an elastic case. For the inelastic case, the only difference is the expression of $T_{3}$ given above, which is obtained numerically as explained below and is slightly lower than the elastic estimate.

At positions deeper in the shock front, it can be necessary to account for the second generation of intermediate particles (population 4). The model can be extended to four subpopulations: supersonic, subsonic, first-generation intermediate particles, and second-generation intermediate particles. The first-generation intermediate particles arise from a collision between supersonic and subsonic particles as previously explained. The difference is that they become second-generation intermediate particles after a second collision with existing subsonic particles and turn into subsonic particles after a third collision. As detailed in Appendix A, the volume fraction of population 4 can be obtained.

The expressions for $\phi_{3}$ and $\phi_{4}$ derived above and in Appendix A need to be slightly corrected to account for the two-dimensionality of the granular flow. The results of this model are plotted as solid lines in Fig. 3 along with the measured volume fractions of these two subpopulations already discussed in Fig. 3. The model shows a good agreement with experimental measurements using particle tracking giving an a posteriori justification for the different assumptions made, such as neglecting collisions between intermediate particles as well as collisions of these intermediate particles with the supersonic stream. The values of $A_{32} / A_{12}$ and $A_{42} / A_{12}$ used to fit our experimental results are, however, different from the calculated value by about $20 \%$. We believe that the non-Gaussian velocity distributions in the granular gas can explain this difference.

\section{The velocity PDF of the intermediate particles}

Now that we can model the volume fraction of the intermediate populations, we need to calculate the velocity PDFs of these intermediate particles after 1, 2, or more collisions. We detail the way we calculate the velocity distributions of these intermediate particles in the following.

From our model, intermediate particles are the result of collisions between supersonic and subsonic particles. PDFs of velocities have to be determined for these two subpopulations. In the shock wave induced by a dilute granular flow around a circular obstacle, the velocity PDF of the supersonic stream can be obtained from the full velocity PDF as the peak at the incident stream velocity: The population of supersonic particles is obtained from the trajectories. A particle is supersonic up to its first shock with a subsonic particle. The subsonic particles have a zero mean velocity and PDFs of parallel and perpendicular velocities that are isotropic, as shown in Fig. 4. By taking a PDF of $v$ for the subsonic particles similar to that of $u$ to which population 1 has been subtracted, one obtains the full velocity PDF of the subsonic population to a good approximation. These experimentally determined distributions are then used to calculate the PDFs of velocities of intermediate particles.

For two hard spheres, with velocities $\vec{c}_{1}$ and $\vec{c}_{2}$ before the collision, which undergo an inelastic collision with friction, we can write the following relations:

$$
\begin{aligned}
\vec{C}_{1}+\vec{C}_{2}= & \vec{c}_{1}+\vec{c}_{2}, \\
\left(\vec{C}_{1}-\vec{C}_{2}\right) \vec{e}_{n}= & -r\left(\vec{c}_{1}-\vec{c}_{2}\right) \vec{e}_{n}, \\
{\left[\left(\vec{C}_{1}-\vec{C}_{2}\right)-\left(\vec{c}_{1}-\vec{c}_{2}\right)\right] \vec{e}_{t}=} & -\mu \mid\left[\left(\vec{C}_{1}-\vec{C}_{2}\right)\right. \\
& \left.-\left(\vec{c}_{1}-\vec{c}_{2}\right)\right] \vec{e}_{n} \mid,
\end{aligned}
$$

where $\vec{C}_{1}$ and $\vec{C}_{2}$ are velocities after the collision, $\overrightarrow{e_{n}}$ a unit vector linking the two sphere centers, $\overrightarrow{e_{t}}$ is a unit vector perpendicular to $\vec{n}$, and $r$ and $\mu$ are, respectively, the inelasticity coefficient and the friction coefficient with constant values $r=0.95$ and $\mu=0.15$ for steel beads. Velocities after 
the collision can be calculated:

$$
\begin{aligned}
\vec{C}_{1}= & \vec{c}_{1}-\frac{1+r}{2}\left[\left(\vec{c}_{1}-\vec{c}_{2}\right) \vec{e}_{n}\right] \vec{e}_{n} \\
& -\frac{\mu(1+r)}{2}\left|\left(\vec{c}_{1}-\vec{c}_{2}\right) \vec{e}_{n}\right| \vec{e}_{t}, \\
\vec{C}_{2}= & \vec{c}_{2}-\frac{1+r}{2}\left[\left(\vec{c}_{2}-\vec{c}_{1}\right) \vec{e}_{n}\right] \vec{e}_{n} \\
& +\frac{\mu(1+r)}{2}\left|\left(\vec{c}_{1}-\vec{c}_{2}\right) \vec{e}_{n}\right| \vec{e}_{t} .
\end{aligned}
$$

Velocities of the first generation intermediate particles are calculated using the velocity distributions of supersonic and subsonic particles and a random impact parameter. For each value of the impact parameter, a large number of supersonic and subsonic velocities with a probability given by the PDFs are selected to ensure the convergence of the calculated distributions (see Appendix A). Similarly, velocities of the second-generation intermediate particles are calculated using the velocities of the first-generation intermediate particles colliding with subsonic particles. The calculated PDFs of the velocities are shown in Fig. 4 and show a good agreement with experimental measurements from particle tracking.

This good agreement confirms that the first generation intermediate particles (population 3) ultimately come from collisions between the subsonic and supersonic populations only and that the second-generation intermediate particles (population 4) come from collisions between population 3 and subsonic particles mostly. By expanding our hypothesis, the third-generation intermediate particles (population 5) only come from binary collisions between population 4 and subsonic particles. The velocity PDFs of these thirdgeneration intermediate particles is again in good agreement with measurements shown in Fig. 4. We have to keep in mind here that these distributions are obtained just after a collision. These PDFs are not necessarily representative of the distribution of the full intermediate population as explained above. Nonetheless, Fig. 5 suggests that the distribution of velocities just after collision is a good approximation of the distribution of the full intermediate population at least after one collision.

Having identified the origin of the intermediate particles, we can calculate their effect on the full velocity PDFs as shown in Fig. 6. Here, the velocity PDFs of the supersonic and subsonic populations are fixed as explained above, and the intermediate particle velocity PDFs can be calculated after the first and the second collisions. Here, and for convenience, the PDFs of the subsonic and supersonic populations were fit to a symmetric functional shape of the form $\exp \left(-\left[u^{2} / v_{0, i}^{2}+\left(v-V_{i}\right)^{2} / v_{0, i}^{2}+w^{2} / v_{0, i}^{2}\right]^{\eta}\right)(i=1,2)$, with $\eta$ obtained from nonlinear curve fitting. This functional shape is not modified for the supersonic population as the distance from the obstacle changes while only $v_{0,2}$ changes for the subsonic population $\left(V_{2}=0\right)$. The value of $\eta$ does not depend on the Mach number and is near 0.45. These functional shapes were then used to generate the intermediate population numerically using a homemade program. A single collision turns out to be almost sufficient to mimic the full PDF of $v$ at different $y$ positions. Including the second-generation intermediate particles (population 4) gives a better fit.

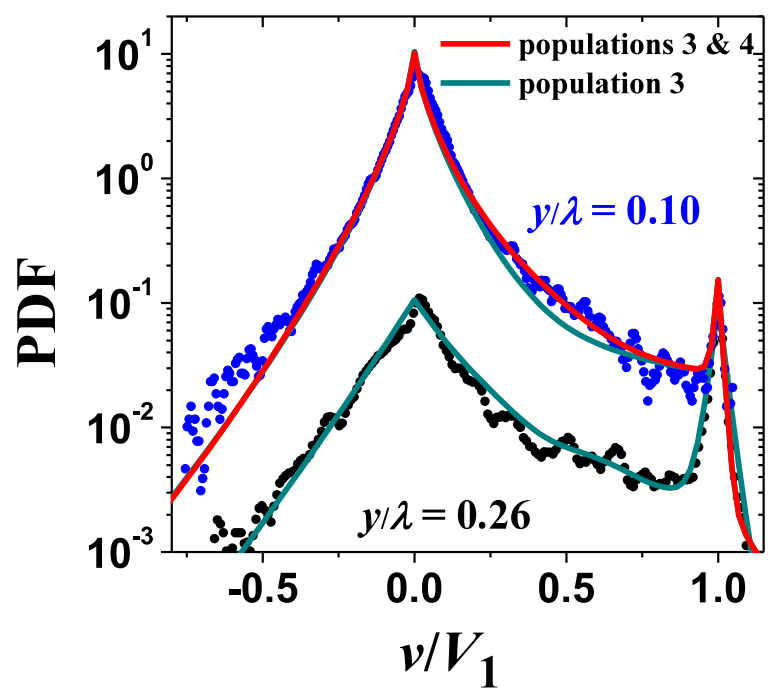

FIG. 6. Velocity PDFs: experimental data and the model for two different $y$ positions. Green lines are fits with first-generation intermediate particles, and red lines with first- and second-generation intermediate particles. In order to improve visibility, the PDF for $y / \lambda=0.26$ is rescaled by a factor $1 / 30 .\left(e=1.5 \mathrm{~mm}, \phi_{1}^{*}=0.007\right.$, $V_{1}=1.1 \mathrm{~m} / \mathrm{s}$, and $M \sim 30$ ).

The values of $\phi_{3}(y)$ and $\phi_{4}(y)$ needed to fit the distributions in Fig. 6 are small, of the order of $\phi_{1}^{*}$, compared to $\phi_{\text {tot }}$ (see Fig. 3 and inset of Fig. 3), which increases up to $30 \phi_{1}^{*}$. Also, these values, shown as the solid symbols in Fig. 3, agree well with the measured $\phi_{3}(y)$ and $\phi_{4}(y)$, already discussed in Fig. 3 above, giving an a posteriori justification of our simple model and the different hypotheses used notably that the distribution of the intermediate populations can be approximated by that just after collision. Note here that despite the fact that our experimental system is quasi-twodimensional, three-dimensional effects have to be considered carefully as shown in Appendix B.

\section{APPLICATION TO SHOCK WAVES IN A VIBRATED GRANULAR GAS AND IN MOLECULAR GASES}

\section{A. The vibrated granular gas}

In this section we extend the above analysis to probe the structure of the velocity distributions in another setup where shock waves are obtained in a vibrated granular gas. Here, a homogeneous granular gas is obtained with steel beads (diameter $d=1 \mathrm{~mm})$ enclosed in a thin horizontal cell $(36 \times$ $21 \times 0.3 \mathrm{~cm})$ vibrated vertically using an electromagnetic shaker with frequencies ranging from 28 to $46 \mathrm{~Hz}$ and an acceleration between 1.5 and $4 \mathrm{~g}$. With a vibration amplitude of $1 \mathrm{~mm}$, we obtain an homogeneous granular gas with a volume

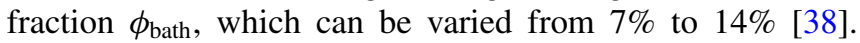
The cell consists of a lower anodized aluminium plate, an upper glass plate, and aluminium spacers of 3-mm thickness. A rectangular obstacle, with 7-cm width and 3-mm thickness, is translated at a constant speed in the granular gas as shown in Fig. 7(a). At speeds greater than $\sim 10 \mathrm{~cm} / \mathrm{s}$, corresponding to the speed of sound in the granular gas, a normal shock wave is generated: beads accumulate, form dense layers near the 

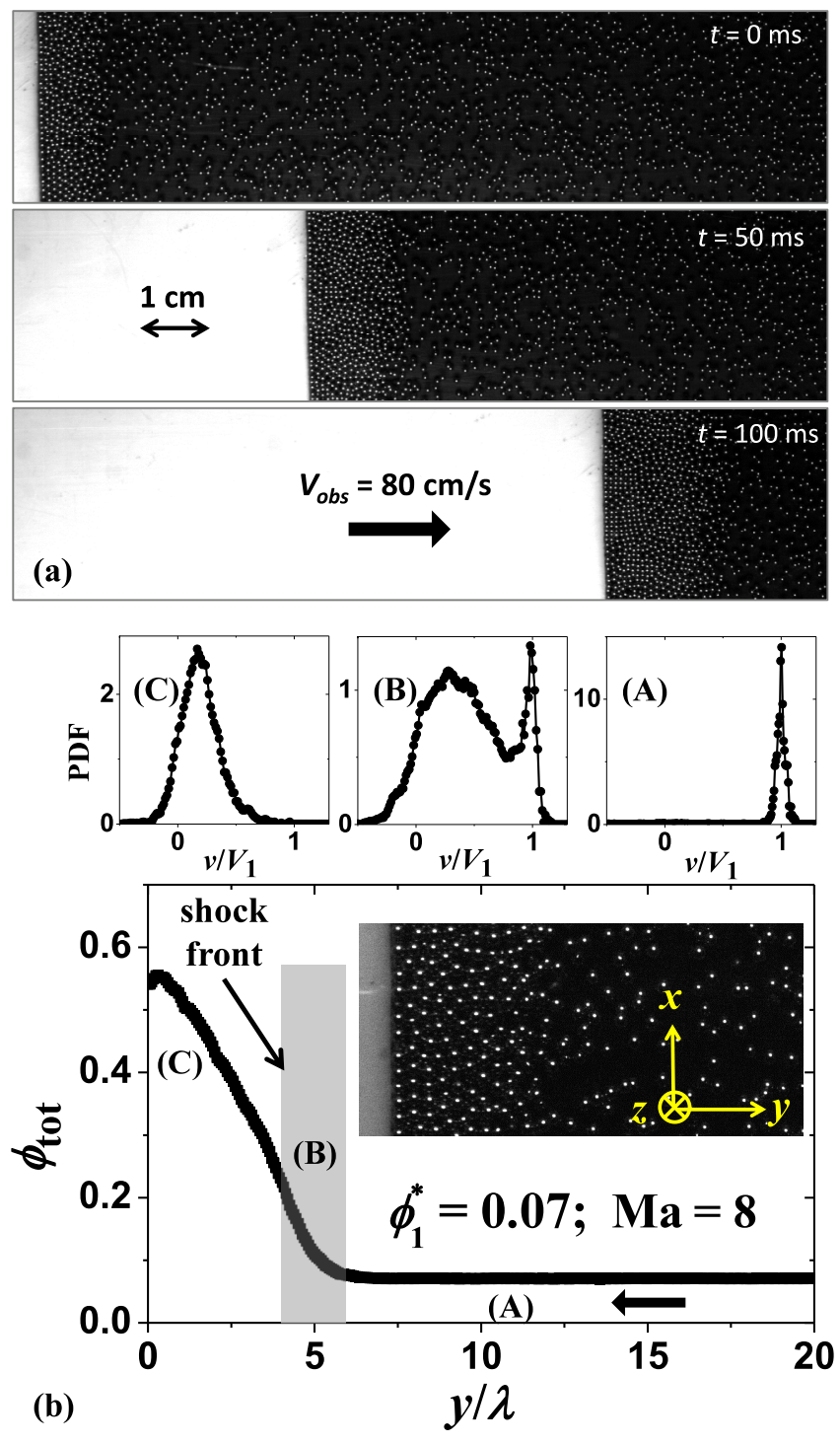

FIG. 7. (a) Photographs of the displacement of a rectangular obstacle (with a velocity $V_{\text {obs }}=80 \mathrm{~cm} / \mathrm{s}$ ) in a vibrated granular gas for $\phi_{1}^{*} \sim 0.07$ and $\mathrm{Ma} \sim 8$. The photographs are taken using reflection from a broad white light source. The contrast is increased to highlight the steel beads. The photographs show the accumulation of particles in the shock front induced by the displacement of the obstacle, which appears light gray in the left part of the image. The obstacle moves from left to right. In the reference frame of the shock front, the supersonic stream is from right to left. (b) Volume fraction profile $\phi_{\text {tot }}$ with PDFs of particle velocities in the reference frame of the shock front, in the three different regions shown in the insets, in a vibrated granular gas for $\phi_{1}^{*} \sim 0.07$ and Ma $\sim 8$. The particle density is large near the obstacle and decreases as the distance to the obstacle increases. Note that this profile is only plotted in the area where the volume fraction does not reach the maximum value near $\phi_{\mathrm{MAX}}$, the random close-packing volume fraction. The photograph shows a zoom of the shock front in the vibrated granular gas.

obstacle, and a well-defined shock front ensues [see Fig. 7(a)]. The rectangular shape of the obstacle used allows only a weak transverse flow so that the flow has a quasi-one-dimensional structure with the shock front normal to the flow direction. The shock propagates with a constant speed $V_{\text {front }}$. In the reference frame of the shock front, the supersonic population is the vibrated gas far from the obstacle which has a velocity $V_{1}=-V_{\text {front }}$. The main interest of this setup is to generate granular shock fronts in a different geometry and with a range of Mach numbers lower than the above experiments and typically between 4 and 20 . The combination of the two experiments thus allows to explore Mach number effects between for 4 and 60 .

Figure 7(b) shows the main features of the shock front in this configuration. Note that the total volume fraction is low far from the obstacle and increases rapidly near the shock front to reach values near 0.5 in the vicinity of the obstacle as has been observed in previous experiments of shock formation in granular gases. Here, the volume fraction was extracted from images of the top layer and corrected for three-dimensional effects using mass conservation as explained in Appendix B. The velocity distributions obtained far from the obstacle show a sharp peak indicative of the supersonic population. The distributions become wider and centered at a smaller value near zero in the vicinity of the obstacle, indicating the presence of the subsonic population. In the front region, however, the distribution looks bimodal and has the signature of the presence of intermediate velocity particles as discussed above and shown in the insets of Fig. 7(b).

The results described in the previous sections of this paper show that the velocity distributions can be well described by a simple model invoking the coexistence of at least three populations in the shock front: a supersonic population, a subsonic population, and a population of first-generation intermediate particles (population 3) coming from single collisions between these two populations. A better description is obtained with a four-population model, but a three-population model is a relatively good approximation as shown in Fig. 6. While in the above experiments using gravity-driven flow around a circular obstacle it was possible to track particles, analyze their collisions, and therefore extract each population separately, it was not possible to directly obtain the velocity distributions and the volume fractions of each population in the vibrated granular gas experiment. Nonetheless, a similar analysis using three populations can be carried out.

In order to analyze these distributions using the model detailed above, we need to obtain the velocity distributions of the supersonic and subsonic populations. The velocity distribution of the supersonic population is obtained from the peak at the incident velocity. The functional shape of this distribution is assumed similar to that far away from the obstacle and can be fit to $\exp \left(-\left[u^{2} / v_{0,1}^{2}+\left(v-V_{1}\right)^{2} / v_{0,1}^{2}+w^{2} / v_{0,1}^{2}\right]^{\eta}\right)$. Here again and as in the previous experiment, the values of $v_{0,1}$ and $\eta$ do not change as the distance to the obstacle varies. The value of $\eta$ does not depend on the Mach number and is near 0.8; the value of $v_{0,1}$, on the other hand, depends on the vibration parameters. The velocity distribution of the component along the flow direction $v$ of subsonic particles is obtained from the PDFs of $u$ with the supersonic particles subtracted. This subtraction consists of removing particles with velocities $v$ greater than a threshold value typically chosen between 0.7 and 0.9 times $V_{1}$ depending on the Mach number. The shape of the PDFs of $u$ did not depend markedly on the exact value of this threshold. We cannot subtract population 3 as it is not known, but in the example above this subtraction does 
not affect the distribution measurably (see Figs. 4 and 5). As explained above, the velocity distributions of $u$ and $v$ are roughly similar for the subsonic population as the subtraction of particles having experienced a few collisions seems to remove the influence of the intermediate particles as shown in Figs. 4 and 5. Note that the mean velocity of the subsonic population $V_{2}$ is not known and is taken as an additional adjustable parameter along with the amplitudes of the PDFs of the supersonic, the subsonic, and the intermediate particles. We include $V_{2}$ in the fit procedure by generating the velocity distributions for a wide range of subsonic mean velocities and the best agreement with experimental measurements is chosen. The PDF of the subsonic population is again fit (for convenience in the numerical calculation) to the functional shape $\exp \left(-\left[u^{2} / v_{0,2}^{2}+\left(v-V_{2}\right)^{2} / v_{0,2}^{2}+w^{2} / v_{0,2}^{2}\right]^{\eta}\right)$, the value of $\eta$ turns out to be 0.8 , while the value of $v_{0,2}$ depends on the Mach number and the position along the shock. Knowing the PDF shape of the supersonic particles and the subsonic particles, the first generation of intermediate particles (population 3) are numerically generated. These three subpopulations are sufficient to fit the full PDF of $v$ as shown in Fig. 8 for different Mach numbers and positions along the shock front. This figure shows the experimental data along with a fit to our model as well as the PDFs of the different populations present. The good agreement between our model and experimental results shows again that the assumptions used are reasonable and that the origin of the intermediate population is single collisions between the two streams independently of the experimental setup used.

\section{B. Molecular gases}

Here, we go further and show that our proposed analysis and model can be extended to shocks in other systems and notably to shocks in molecular gases. As we have mentioned in the Introduction, measurements of the velocity distributions in molecular gases show the presence of a substantial proportion of particles with intermediate velocities and which are not described correctly by using a 2 population model. We here examine whether a three population model as proposed above for granular shocks can be applied to this case. In order to
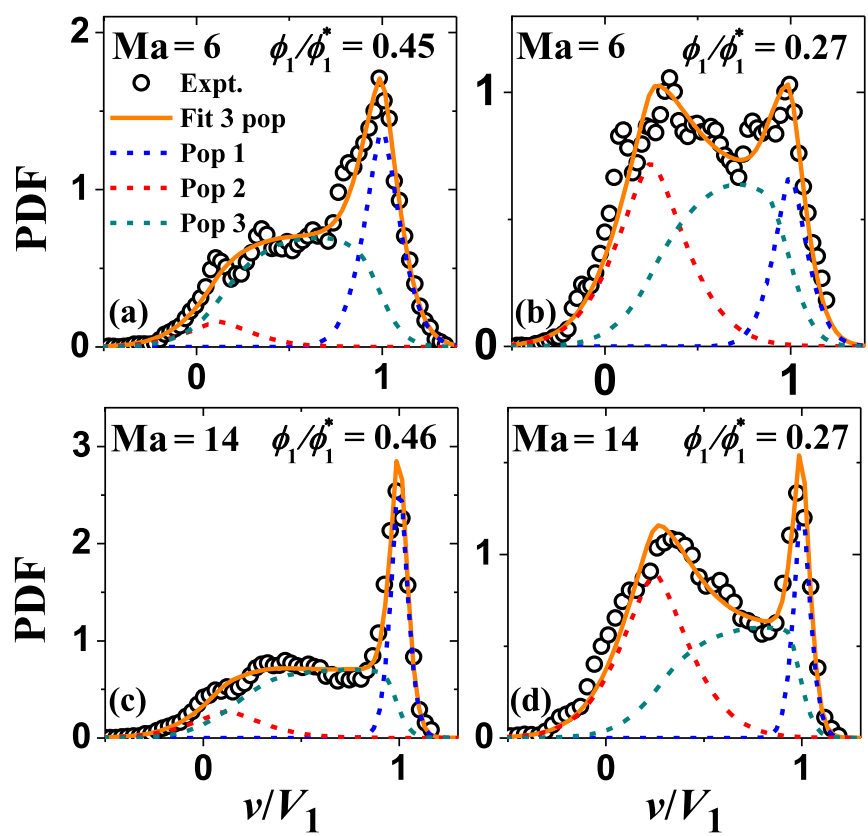

FIG. 8. Experimental PDFs of $v$ for (a) $\phi_{1} / \phi_{1}^{*}=0.45$ and (b) $\phi_{1} / \phi_{1}^{*}=0.27$ in the vibrated granular gas at $\mathrm{Ma}=6$ with the decomposition into the supersonic particles, the subsonic particles, and the intermediate particles. (c), (d) the same at $\mathrm{Ma}=14$. Note that the subsonic population PDF has a peak at a nonzero velocity given by $V_{2}$.

do so, we carry out a similar analysis of velocity distributions obtained from experimental [5,6] and numerical studies [27] on shock waves in gases. In molecular gases, we assume that the supersonic particles have Gaussian velocity distributions with known temperature $T_{1}$ and mean velocity $V_{1}$ but that the subsonic particles have Gaussian velocity distributions with unknown temperature $T_{2}$ and mean velocity $V_{2}$. Linking these two parameters is necessary to generate velocity distributions of intermediate particles. We first use the Euler equations in molecular gases with supersonic, subsonic, and intermediate particles (respectively, noted 1, 2, and 3) to obtain

$$
\begin{aligned}
\rho_{1} V_{1}+\rho_{2} V_{2}+\rho_{3} V_{3} & =\rho_{1}^{*} V_{1}, \\
\rho_{1}\left(V_{1}^{2}+\frac{k}{m} T_{1}\right)+\rho_{2}\left(V_{2}^{2}+\frac{k}{m} T_{2}\right)+\rho_{3}\left(V_{3}^{2}+\frac{k}{m} T_{3}\right) & =\rho_{1}^{*}\left(V_{1}^{2}+\frac{k}{m} T_{1}\right), \\
\rho_{1} V_{1}\left(V_{1}^{2}+(n+2) \frac{k}{m} T_{1}\right)+\rho_{2} V_{2}\left(V_{2}^{2}+(n+2) \frac{k}{m} T_{2}\right)+\rho_{3} V_{3}\left(V_{3}^{2}+(n+2) \frac{k}{m} T_{3}\right) & =\rho_{1}^{*} V_{1}\left(V_{1}^{2}+(n+2) \frac{k}{m} T_{1}\right) .
\end{aligned}
$$

These equations and the following equations $V_{3}=$ $\frac{V_{1}+V_{2}}{2}, \quad T_{3}=\frac{T_{1}+T_{2}}{2}+\frac{m\left(V_{1}-V_{2}\right)^{2}}{4 n k}$, which are the equivalent of Eqs. (14) for molecular gases, impose a relation between the temperature $T_{2}$ and mean velocity $V_{2}$ of subsonic particles. We have then used the assumptions explained above to generate the PDFs of the subsonic and supersonic populations from the measured PDFs and used them to calculate the PDF of the intermediate particles (for molecular gases we use $r=0$ and $\mu=0$ ). The adjustable parameters are the amplitudes of the PDFs and the value of $V_{2}$.

We find that the first generation intermediate particles are sufficient to fit the full velocity PDF in gases for different positions in the shock front as well as different Mach numbers as shown in Fig. 9. Here data has been extracted from experiments as well as numerical simulations, which are both well modeled by our analysis, suggesting that single collisions 

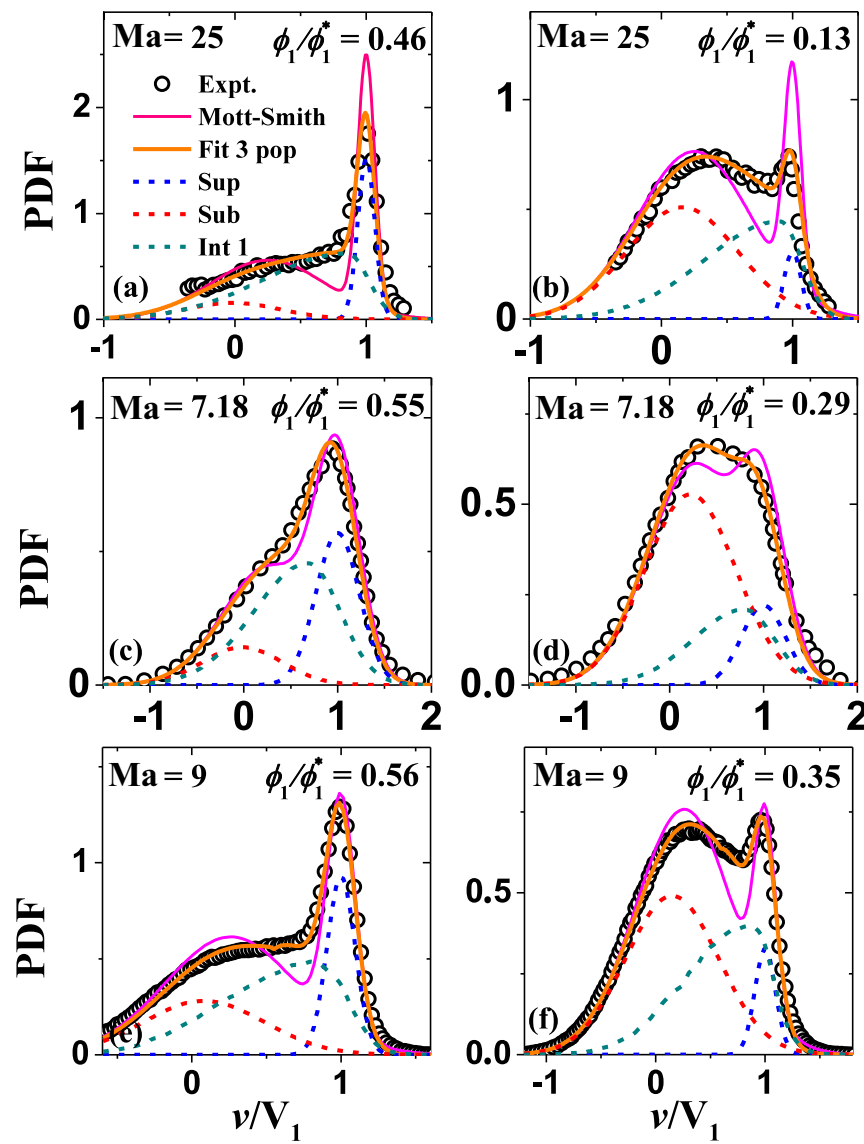

FIG. 9. (a), (b) Experimental measurements from Ref. [6] for $\phi_{1} / \phi_{1}^{*}=0.46$ and $\phi_{1} / \phi_{1}^{*}=0.13$ at $M=25$ with the PDFs from Mott-Smith theory and our three-population model. (c), (d) The same from Ref. [5] at Ma=7.18. (e), (f) The same from Ref. [27] at $\mathrm{Ma}=9$. Note that the subsonic population PDF has a peak at a nonzero velocity given by $V_{2}$.

between the supersonic and subsonic streams are sufficient to account for the intermediate population. Our model allows a much better agreement with experimental data as well as data obtained in numerical simulations than the distributions with only two populations considered in the Mott-Smith theory.

\section{CONCLUSION}

In the present paper, we have attempted to model the probability distribution functions of the velocity across a shock front. We have used a model system consisting of a granular gas flowing past an obstacle to illustrate that the key to understanding these distributions is the role of collisions in generating intermediate velocities. Previous models of the shock front which split the contributions to the velocity distribution into two main contributions coming from a supersonic stream and a subsonic stream turn out to be incomplete. A substantial portion of the measured velocities emanates from contributions not represented by these two streams alone. Our experiments and the focus on particle collisions in the shock front show that these two streams interact with each other as expected and generate particles with intermediate velocities. These experiments also show that these particles are for the most part produced by single binary collisions between the two streams. Taking into account the contribution of second generation intermediate particles emanating from the collisions between the first generation intermediate particles and the subsonic stream turns out to give better agreement with measured velocity distributions but their importance is minor. We have proposed a simple model to calculate the contribution of these intermediate particles to the velocity distributions which conforts these observations. This simple model relies on the measured PDFs to extract the contribution of the two primary streams (the subsonic and supersonic one) and uses this information to generate the missing intermediate population by calculating the effects of binary collisions between the two main streams on the velocity PDF. This analysis has been shown to work reasonably well for granular shocks around an obstacle where this procedure is validated by direct measurements of the intermediate populations through particle tracking. It has also been used to explain the velocity distributions in two other cases, shocks in vibrated granular gases and shocks in molecular gases, for which it is not possible to measure the contribution of intermediate particles directly. In both of these latter cases, the contribution of a single additional population of intermediate particles turns out to be sufficient to reproduce the shape of the full velocity profile. This additional population is obtained by calculating the result of single binary collisions between the two main streams. In the absence of a model for the full distribution, our semiempirical attempt is one of the few to give very reasonable agreement with measured velocity PDFs across a shock front in different systems. Further, and since only a few collisions are sufficient to capture the full PDF, our insight may be useful in constructing approximate models.

To summarize, from shock waves induced in granular gases for two different setups, we propose a new description for the velocity distributions, which consists of a superposition of an incident supersonic stream, a subsonic stream, and a third intermediate state. This intermediate state consists mostly of particles having experienced one or two collisions only rendering the prediction of the full velocity distribution relatively simple. This analysis is applied to dilute granular shocks as well as shocks in gases.

\section{ACKNOWLEDGMENTS}

We thank E. Maillard and L. Haelman from the Machine shop for help with the two experimental setups. A. Vilquin was supported by a fellowship of the University of Bordeaux.

\section{APPENDIX A: CALCULATION OF THE VOLUME FRACTION AND VELOCITY DISTRIBUTION OF SECOND-GENERATION INTERMEDIATE PARTICLES}

At positions deeper in the shock front, it can be necessary to take into account second-generation intermediate particles. The model can be extended to include four sub-populations: supersonic, subsonic, first-, and second-generation intermediate particles, respectively noted with index $i$ of 1, 2, 3, and 4 . First-generation intermediate particles arise from a collision between supersonic and subsonic particles as previously 
explained. The difference is that they become secondgeneration intermediate particles after a second collision with existing subsonic particles and turn into subsonic particles after a third collision. Appearance and disappearance processes can be described by the following equations:

$$
\begin{aligned}
& \frac{\partial\left(\phi_{1} V_{1}\right)}{\partial y}=-J_{12} \\
& \frac{\partial\left(\phi_{2} V_{2}\right)}{\partial y}=-J_{12}-J_{32}+J_{42}, \\
& \frac{\partial\left(\phi_{3} V_{3}\right)}{\partial y}=2 J_{12}-J_{32} \\
& \frac{\partial\left(\phi_{4} V_{4}\right)}{\partial y}=2 J_{32}-J_{42} .
\end{aligned}
$$

Because of momentum and energy conservation, the mean velocity and the temperature of intermediate particles (1) and (2) can be expressed

$$
\begin{array}{ll}
V_{3}=\frac{V_{1}+V_{2}}{2} & T_{3}=\frac{T_{1}+T_{2}}{2}+\frac{\left(V_{1}-V_{2}\right)^{2}}{4 N}, \\
V_{4}=\frac{V_{3}+V_{2}}{2} & T_{4}=\frac{T_{3}+T_{2}}{2}+\frac{\left(V_{3}-V_{2}\right)^{2}}{4 N} .
\end{array}
$$

The volume fraction of first-generation intermediate particles is again obtained from Eq. (18) and the expression in Eq. (19). From Eqs. (A1), (A2), (A4), and (8), the volume fraction of second-generation intermediate particles can be obtained from

$$
\begin{aligned}
\frac{\partial\left(\phi_{4} v_{4}\right)}{\partial\left(\phi_{1} v_{1}\right)} & =-4-2 \frac{\partial\left(\phi_{3} v_{3}\right)}{\partial\left(\phi_{1} v_{1}\right)}+\frac{\phi_{4}}{\phi_{1}} \frac{v_{4}-v_{2}}{v_{1}-v_{2}} \frac{A_{42}}{A_{12}} \\
& =-\frac{A_{32}}{A_{12}} \frac{\phi_{3}}{\phi_{1}}+\frac{1}{4} \frac{A_{42}}{A_{12}} \frac{\phi_{4}}{\phi_{1}} .
\end{aligned}
$$

The volume fraction of the first-generation intermediate particles is again given by Eq. (19), and we can express the volume fraction of the second-generation intermediate particles as

$$
\begin{aligned}
\phi_{3} & =K_{1} \phi_{1}^{*}\left[\frac{\phi_{1}}{\phi_{1}^{*}}-\left(\frac{\phi_{1}}{\phi_{1}^{*}}\right)^{\alpha}\right], \quad K_{1}^{-1}=\frac{1}{2} \frac{V_{3}}{V_{1}}(\alpha-1), \\
\alpha & =\frac{1}{2} \frac{A_{32}}{A_{12}} \frac{V_{1}}{V_{3}}, \\
\phi_{4} & =K_{2} \phi_{1}^{*}\left[\frac{\frac{\phi_{1}}{\phi_{1}^{*}}-\left(\frac{\phi_{1}}{\phi_{1}^{*}}\right)^{\beta}}{\beta-1}+\frac{\left(\frac{\phi_{1}}{\phi_{1}^{*}}\right)^{\beta}-\left(\frac{\phi_{1}}{\phi_{1}^{*}}\right)^{\alpha}}{\beta-\alpha}\right], \\
K_{2}^{-1} & =\frac{1}{4} \frac{V_{4}}{V_{1}} \frac{(\alpha-1)}{\alpha}, \quad \beta=\frac{1}{4} \frac{A_{42}}{A_{12}} \frac{V_{1}}{V_{4}} .
\end{aligned}
$$

These two expressions are used in Fig. 3 of the main text with corrections due to the two-dimensional nature of the gravity driven granular flow around the circular obstacle.

Let us describe the procedure to obtain the velocity PDF of the first-generation intermediate particles $\mathrm{PDF}_{3}(\vec{c})$. Consider a shock between a supersonic particle and a subsonic particle with a velocity $\overrightarrow{c_{1}}$ and $\overrightarrow{c_{2}}$, respectively, and an impact point $\left(x_{1}, y_{1}, z_{1}\right)$ with the constraint $\sqrt{x_{1}^{2}+y_{1}^{2}+z_{1}^{2}}=d / 2$ and with a uniform probability $p$ in the plane $\left(x_{1}, z_{1}\right)$. The center of the subsonic particle is at $(0,0,0)$. With the impact point, we can calculate $\overrightarrow{e_{n}} \sim\left(x \overrightarrow{e_{x}}+y \overrightarrow{e_{y}}+z \overrightarrow{e_{z}}\right), \overrightarrow{e_{t}} \sim$ $\left[\left(\overrightarrow{c_{1}}-\overrightarrow{c_{2}}\right) \times \overrightarrow{e_{n}}\right] \times \overrightarrow{e_{n}}$, and the velocity of both intermediate particles after the shock with a velocity denoted $\overrightarrow{c_{3}}=\overrightarrow{C_{1}}$ or $\overrightarrow{C_{2}}$ (see the collision rules, Sec. III C). The values of $\overrightarrow{c_{1}}, \overrightarrow{c_{2}}$, and $\left(x_{1}, z_{1}\right)$ are randomly generated. Each calculated velocity $\overrightarrow{c_{3}}$ for each impact position contributes to the PDF of the velocity of the first-generation intermediate particles $\mathrm{PDF}_{3}(\vec{c})$ with a weight given by $\operatorname{PDF}_{1}\left(\overrightarrow{c_{1}}\right) \operatorname{PDF}_{2}\left(\overrightarrow{c_{2}}\right) p / 2\left|\overrightarrow{c_{1}}-\overrightarrow{c_{2}}\right|$. For the granular gas, collisions are inelastic, and $\operatorname{PDF}_{1}(\vec{c})$ and $\operatorname{PDF}_{2}(\vec{c})$ (for the supersonic and subsonic populations respectively) are obtained from experimental measurements as detailed in the text $\left(\operatorname{PDF}_{i}(\vec{c})=\right.$ $\exp \left(-\left[u^{2} / v_{0, i}^{2}+\left(v-V_{i}\right)^{2} / v_{0, i}^{2}+w^{2} / v_{0, i}^{2}\right]^{\eta}\right), \quad i=1,2, \quad \eta<$ $1)$. For molecular gases, collisions are elastic (the collisions rules are used with $r=1$ and $\mu=0)$ and $\operatorname{PDF}_{1}(\vec{c})$ and $\mathrm{PDF}_{2}(\vec{c})$ are assumed Gaussian $\left(\mathrm{PDF}_{i}(\vec{c})=\exp (-m(\vec{c}-\right.$ $\left.\left.\left.V_{i} \overrightarrow{e_{y}}\right)^{2} / 2 k T_{i}\right), i=1,2\right)$. The $\mathrm{PDF}_{3}(\vec{c})$ of intermediate particles 3 is then obtained numerically using $10^{6}$ trials.

Note that the velocity components $u, v$, and $w$ of the supersonic particles are assumed to have the same PDF shapes and widths but not the same mean values while the $u, v$, and $w$ components of the subsonic stream have equivalent PDFs. This has been tested experimentally for the flow around an obstacle as well as the granular gas but is assumed for the molecular gases. A major assumption used here is that the first-generation intermediate particle distribution can be approximated by that obtained just after collision, which amounts to neglecting the effect of advection and disappearance of these particles. While this has been tested in the flow around an obstacle where a reasonable agreement was found between the intermediate population calculated using this assumption and the full population, we have used this assumption without justification for the vibrated granular gas as well as molecular gases. The good agreement found a posteriori conforts us in the use of this assumption, which renders the calculation possible. Without this assumption, it is very difficult to estimate the PDFs of this population.

The procedure to calculate velocities of the secondgeneration intermediate particles is similar to the procedure to generate the velocities of the first generation intermediate particles, except that the velocity distribution of supersonic particles is replaced by the velocity distribution of the first generation intermediate particles.

\section{APPENDIX B: INFLUENCE OF THE THIRD DIMENSION}

Although the experiments presented in this work are quasitwo-dimensional, the particle motion is three-dimensional. For gases, the experiments are three-dimensional. In this latter case, the velocity distributions along the $z$ axis are assumed to be those following the $x$ axis, which is justified by the symmetry of the flows. For the granular experiments presented in this work, the $x z$ symmetry is broken by the presence of the walls. So it raises the question of particle motion in the third dimension and its influence. In our experiments, the movement of particles along the $z$ axis was measured and its contribution was included in the description.

We quote below a number of problems posed by the presence of walls and the solutions we have brought to resolve these issues in the context of the analysis presented in this work. 


\section{The determination of the volume fraction}

In the granular horizontal flow experiment, imaging does not allow us to detect all the particles leading to an underestimation of the local volume fraction of the granular flow. We obtain the true volume fraction $\phi_{\text {tot }}$ using the following procedure: When the obstacle starts moving, the detected density profiles show rapidly a translation invariance. In the reference frame of the shock front, the flow is stationary. Stationary of the flow allows us to obtain the true volume fraction by the following conservation equation: $\frac{\partial}{\partial y}\left(\phi_{\text {tot }} V\right)+\phi_{\text {tot }} \frac{\partial}{\partial x}(U)=0$, where $V$ and $U$ are the measured mean velocities along the propagation axis $y$ and the transverse $x$ axis respectively in the reference frame of the shock front. $\phi_{\text {tot }}$ can be obtained numerically from the following expression:

$$
\phi_{\mathrm{tot}}(y)=\phi_{1}^{*} \exp \left(\int_{y}^{+\infty}\left[\frac{\partial V}{\partial y}+\frac{\partial U}{\partial x}\right] \frac{d y}{V}\right)
$$

\section{Particle motion in the third dimension}

We have access to the movement of particles in the $z$ direction by two experimental methods. The first method is to film the granular flows at an angle $\theta=50^{\circ}$ relative to the $z$ axis, which gives access to particle movement and velocity distributions in the direction $\cos (\theta) \overrightarrow{e_{x}}+\sin (\theta) \overrightarrow{e_{z}}$. Velocity distributions along $\overrightarrow{e_{x}}$ are known so the velocity distributions along $\overrightarrow{e_{z}}$ can be estimated by deconvolution. The second method is to slightly defocus the camera (with $\theta=0$ ). Then the intensity of the reflection from the particle varies with $z$. By calibrating this intensity versus $z$, this method gives access to the position of the particle along $z$. Using this second method, we have access to positions of particles in the three spatial directions. However, it is less accurate along $z$. This analysis also shows that the particles have many collisions with the walls. The influence of these walls will be discussed below.

Particle motion along $z$ is different in the two granular experiments discussed in this paper. For the vertical flow experiment, the velocity distribution for all particles along $z$ is very similar to that obtained along $x$. As the form of these distributions is mainly determined by the population of subsonic particles, it can be concluded that the subsonic particles have isotropic distributions along the three spatial directions.

For the horizontal flow experiment, the movement along $z$ is very different. All particles (in the bath or in the shock front) have a sinusoidal movement very close to the vibrated walls. In the bath, velocity distributions along $z$ (having removed the sinusoidal motion) are wider than those obtained along $x$ (typically 40\%). This is much less significant in the shock front and it can be argued that the subsonic particles have almost identical agitation in the three spatial directions.

Although collisions with the walls are very dissipative, velocity distributions of subsonic particles are broadly isotropic. Presumably the collisions between particles distribute energy evenly between the different spatial degrees of freedom.

\section{The influence of the walls on the velocity distributions of intermediate particles}

The preceding analysis is silent on the intermediate particles. And in fact, the presence of the walls has an influence on the velocity distribution of first-generation intermediate particles. This influence is understood and is included in the description for the vertical experiment. The major effect of the walls is to confine the particles. The main observation is that the velocity distributions of first-generation intermediate particles depend on the distance between the walls. The explanation is the following: The impact parameter is spatially limited by the presence of the walls. In fact, in the numerical calculation of velocity distributions of intermediate particles, we include this effect by limiting spatially the positions of particles at impact. This method allows us to reproduce the evolution of the velocity distributions as a function of the distance between walls. It is noteworthy that this effect is relatively small for $e>2 d$, but it has a significant role for $e=1.5 d$.

Consider a first-generation intermediate particle, it may collide with the walls while remaining intermediate, that is to say, it did not collide with another particle. However, the velocity of this intermediate particle changes during its propagation. This is detectable on the trajectories. The consequence for the description is that the velocity distributions of the intermediate particles at a given position in the shock wave are not quite similar to those measured immediately after impact (Fig. 4). In the analysis (Figs. 5 and 6), the results are slightly better by using the full measured distributions instead of the calculated distributions right after impact.

This analysis could not be made for the horizontal flow experiment since we have no direct access to intermediate particles. For supersonic particles (bath), it was noted that the velocity distributions along $z$ are wider, which should have an influence on the determination of the intermediate particles. We tested numerically the influence of the width of this distribution in the determinations of the distribution of these intermediate particles. This influence is weak, the shape of the distributions of intermediate particles is essentially determined by the eventual confinement and average speeds of each population.
[1] J. D. Anderson, Modern Compressible Flow: With Historical Perspective (McGraw-Hill, New York, 1990).

[2] R. E. Center, Phys. Fluids 10, 1777 (1967).

[3] B. Schmidt, J. Fluid Mech. 39, 361 (1969).

[4] H. Alsmeyer, J. Fluid Mech. 74, 497 (1976).

[5] T. Holtz and E. P. Muntz, Phys. Fluids 26, 2425 (1983).

[6] G. Pham-Van-Diep, D. Erwin, and E. Muntz, Science 245, 624 (1989).
[7] G. C. Pham-Van-Diep, D. A. Erwin, and E. P. Muntz, J. Fluid Mech. 232, 403 (1991).

[8] A. Ramos, B. Maté, G. Tejeda, J. M. Fernández, and S. Montero, Phys. Rev. E 62, 4940 (2000).

[9] S. Mazouffre, P. Vankan, R. Engeln, and D. C. Schram, Phys. Rev. E 64, 066405 (2001).

[10] P. Vankan, S. Mazouffre, R. Engeln, and D. C. Schram, Phys. Plasmas 12, 102303 (2005). 
[11] N. P. Oxtoby, E. J. Griffith, C. Durniak, J. F. Ralph, and D. Samsonov, Phys. Rev. Lett. 111, 015002 (2013).

[12] Y. Amarouchene, J. F. Boudet, and H. Kellay, Phys. Rev. Lett. 86, 4286 (2001)

[13] E. C. Rericha, C. Bizon, M. D. Shattuck, and H. L. Swinney, Phys. Rev. Lett. 88, 014302 (2001).

[14] Y. Amarouchene and H. Kellay, Phys. Fluids 18, 031707 (2006).

[15] K. Huang, G. Miao, P. Zhang, Y. Yun, and R. Wei, Phys. Rev. E 73, 041302 (2006).

[16] A. Levy and M. Sayed, Phys. Fluids 19, 023302 (2007).

[17] J. F. Boudet, Y. Amarouchene, and H. Kellay, Phys. Rev. Lett. 101, 254503 (2008).

[18] N. Sirmas, M. Tudorache, J. Barahona, and M. I. Radulescu, Shock Waves 22, 237 (2012).

[19] N. Sirmas and M. I. Radulescu, Phys. Rev. E 91, 023003 (2015).

[20] H. M. Mott-Smith, Phys. Rev. 82, 885 (1951).

[21] H. Grad, Commun. Pure Appl. Math. 5, 257 (1952).

[22] M. Al-Ghoul and B. C. Eu, Phys. Rev. Lett. 86, 4294 (2001).

[23] M. A. Solovchuk and T. W. H. Sheu, Phys. Rev. E 83, 026301 (2011).

[24] H. Salwen, C. E. Grosch, and S. Ziering, Phys. Fluids 7, 180 (1964).
[25] S. H. Radin, Phys. Fluids 9, 1621 (1966).

[26] A. G. Bashkirov and A. V. Orlov, Phys. Rev. E 53, R17 (1996).

[27] P. Valentini and E. T. Schwartzentruber, Phys. Fluids 21, 066101 (2009).

[28] S. Liu and C. Zhong, Phys. Rev. E 89, 033306 (2014).

[29] S. Ogawa, Proceedings U.S.-Japan Seminar on ContinuumMechanical and Statistical Approaches in the Mechanics of Granular Materials (1978), pp. 208-217.

[30] S. B. Savage and D. J. Jeffrey, J. Fluid Mech. 110, 255 (1981).

[31] P. K. Haff, J. Fluid Mech. 134, 401 (1983).

[32] C. K. K. Lun, S. B. Savage, D. J. Jeffrey, and N. Chepurniy, J. Fluid Mech. 140, 223 (1984).

[33] A. Goldshtein and M. Shapiro, J. Fluid Mech. 282, 75 (1995)

[34] E. L. Grossman, Tong Zhou, and E. Ben-Naim, Phys. Rev. E 55, 4200 (1997).

[35] N. Brilliantov and T. Pöschel, Phys. Rev. E 67, 061304 (2003).

[36] B. Andreotti, Y. Forterre, and O. Pouliquen, Les milieux granulaires: Entre fluide et solide (EDP Sciences, Les Ulis, 2012).

[37] S. B. Savage, J. Fluid Mech. 194, 457 (1988).

[38] K. Roeller, J. P. D. Clewett, R. M. Bowley, S. Herminghaus, and M. R. Swift, Phys. Rev. Lett. 107, 048002 (2011). 